OPEN ACCESS

Edited by:

Imtiaz Ahmad Siddiqui,

University of Colorado Anschutz Medical Campus, United States

Reviewed by:

Zohreh Sanaat,

Tabriz University of Medical

Sciences, Iran

Hamidullah Khan,

University of Wisconsin-Madison,

United States

*Correspondence:

Yinhua Yu

yin_hu_a@hotmail.com

Hongbo Zhao

zhaohongbo01@sina.com

tThese authors have contributed equally to this work

Specialty section:

This article was submitted to Cancer Epidemiology and Prevention,

a section of the journal

Frontiers in Oncology

Received: 31 May 2019 Accepted: 07 August 2019

Published: 21 August 2019

Citation:

Zhou J, Du Y, Lu Y, Luan B, Xu C, Yu Y and Zhao H (2019) CD44 Expression Predicts Prognosis of Ovarian Cancer Patients Through Promoting Epithelial-Mesenchymal Transition (EMT) by Regulating Snail, ZEB1, and Caveolin-1. Front. Oncol. 9:802 doi: 10.3389/fonc.2019.00802

\section{CD44 Expression Predicts Prognosis of Ovarian Cancer Patients Through Promoting Epithelial-Mesenchymal Transition (EMT) by Regulating Snail, ZEB1, and Caveolin-1}

\author{
Jiayi Zhou ${ }^{1,2,3 \dagger}$, Yan Du ${ }^{1,2,3 t}$, Yiling Lu ${ }^{4}$, Baoxin Luan ${ }^{1,2,3}$, Congjian $X u^{1,2,3}$, Yinhua Yu ${ }^{1,2,3 *}$ \\ and Hongbo Zhao ${ }^{1,2,3 *}$
}

1 Obstetrics and Gynecology Hospital, Fudan University, Shanghai, China, ${ }^{2}$ Department of Obstetrics and Gynecology of Shanghai Medical School, Fudan University, Shanghai, China, ${ }^{3}$ Shanghai Key Laboratory of Female Reproductive Endocrine Related Diseases, Shanghai, China, ${ }^{4}$ Department of Systems Biology, University of Texas MD Anderson Cancer Center, Houston, TX, United States

Objectives: CD44, a transmembrane glycoprotein, is involved in the generation of a stem cell niche and maintaining stem cell quiescence. The aim of this study was to evaluate its contribution to ovarian cancer prognosis and progression, as well as explore the possible mechanisms.

Materials and Methods: The expression of CD44 in tissue microarray of 90 ovarian cancer patients was detected by immunohistochemistry. Kaplan-Meier method and Cox proportional hazard model were used to evaluate the factors associated with 5-year overall survival and disease-free survival. CD44 was knocked down by small interfering RNA, the expression of Snail, ZEB1, and Caveolin-1 in a stable Snail-expressing ovarian cancer cell line HO8910PM-Snail (HOPM-Snail) and its control cell line H08910PM-vector (HOPM) was detected by western blotting analysis. Cell clone formation, migration, and invasion of HOPM-Snail and HOPM cells with CD44 silencing were examined by 3-D culture assay, wound healing assay, and transwell assay, respectively.

Results: Over-expression of CD44 was associated with advanced histological grade $(p=0.014)$ and FIGO stage $(p=0.001)$. Multivariate analysis showed that CD44 expression was an independent prognostic factor to predict both overall survival $(p=0.004)$ and disease-free survival $(p=0.025)$ of ovarian cancer patients. Down-regulation of CD44 expression by small silencing RNA abrogated both basal Snail expression and TGF- $\beta 1$-induced Snail expression in HOPM and HOPM-Snail cells. In addition, CD44 knockdown caused a decrease in ZEB1 expression. RPPA data indicated that Caveolin-1 may be another regulative target of CD44, and western blotting analysis confirmed that CD44 knockdown caused an increase in Caveolin-1 expression. However, there was no noticeable reciprocal regulation among ZEB1, Caveolin-1, and Snail. Moreover, CD44 knockdown caused a decrease in cell clone formation, migration, and invasion of HOPM and HOPM-Snail cells. 
Conclusions: As both Snail and ZEB1 are crucial inducers of epithelial-to-mesenchymal transition (EMT), our data suggested that CD44 may be crucial for the EMT process of ovarian cancer. Therefore, CD44 may be a potential prognostic marker as well as treatment target for ovarian cancer.

Keywords: CD44, expression, ovarian cancer, epithelial-to-mesenchymal transition, prognosis, survival

\section{INTRODUCTION}

Ovarian cancer is one of the most common gynecologic cancer and a fatal malignancy in women worldwide (1). There were estimated 295,414 new cases of and 184,799 deaths caused by ovarian cancer in 2018 (1). With an aging population, ovarian cancer is and will continue to be a huge health burden in China (2). Epidemiological studies have identified several risk factors including menstrual and reproductive factors, obesity, hormone therapy, personal history of breast cancer, family history, and genetic mutations (BRCA1 and BRCA2 mutations) $(3,4)$. However, the etiology of ovarian cancer is still unclear. Despite treatment advancement in recent decades, the prognosis of ovarian cancer patients remains poor, with a reported 5-year survival rate of $45 \%$ in the United States (5). Therefore, it is of vital importance to identify the predictive markers of recurrence risk and survival, as well as therapeutic targets.

CD44, as a transmembrane glycoprotein, is involved in the generation and maintenance of the stem cell niche and selfrenewal potential (6). It has been well-documented that high expression of CD44 predicts poor prognosis of various tumors including breast, brain, colon, pancreatic, and gastric tumors, indicating that CD44 may be a valuable prognostic marker and therapeutic target for cancers (7). Although the association of CD44 expression with the survival of ovarian cancer patients has been widely investigated, the role of CD44 in the prognosis of ovarian cancer remains controversial (8-18). Some studies found that increased expression of CD44 closely correlated with poor prognosis of ovarian cancer (8-12). On the contrary, other studies reported that CD44 was not an independent predictor of survival and prognosis (13-18).

Ovarian cancer frequently consists of heterogeneous subpopulations. Among these cell populations, cancer stem cells (CSCs) have been widely accepted to endow ovarian cancer with tumor initiation and self-renewal potential $(19,20)$. CD44, as the most frequently reported CSC marker, is widely used to distinguish CSCs from other populations of cancer cells (21-23). CD44, as well as other CSC markers endoglin (CD105) and CD106 has been proved to be highly expressed in chemo-resistant ovarian cancer cells and in advanced-stage epithelial ovarian cancer tissues, suggesting CD44 may accelerate the progression of ovarian cancer by modulating the properties of CSCs (24).

Epithelial-to-mesenchymal transition (EMT), which enables the invasion of epithelial carcinoma cells to the underlying stroma, is a critical pathophysiological process in epithelial cancer (25). EMT is induced and maintained by critical genes including N-cadherin, Snail, Slug, Twist, Vimentin, and Zinc finger E-box-binding homobox 1 (ZEB1) (26-28). Previous data have shown that CD44 over-expression caused a significant up-regulation of the mesenchymal markers $\mathrm{N}$-cadherin and Vimentin with a concomitant down-regulation of the epithelial markers E-cadherin and Claudin 7 in PA1 and SKOV3 ovarian cancer cells, indicating the possible involvement of CD44 in EMT (29). However, the exact role of CD44 in EMT in ovarian cancer remains elusive and requires further investigation.

In the present study, we systematically evaluated the prognostic value of CD44 in ovarian cancer patients, and explored the modulation of CD44 on EMT in ovarian cancer cell lines. Our study has proposed a new mechanism by which CD44 induces the EMT progress via ZEB1 and Snail in ovarian cancer. Therefore, CD44 may be a potential target for ovarian cancer not only due to its effect on stem cell properties, but also due to its pivotal role in EMT.

\section{MATERIALS AND METHODS}

\section{Study Patients}

This is a retrospective cohort study. We included patients with newly diagnosed sporadic epithelial ovarian cancer at the Obstetrics and Gynecology Hospital of Fudan University in Shanghai, China from May 2006 to November 2008. Specimens were collected by the Tissue Bank of the hospital. Inclusion Criteria were: (1) patients with newly diagnosed sporadic epithelial ovarian cancer, (2) written informed consent was obtained. Exclusion Criteria were: (1) patients with history of other malignant tumors, (2) did not receive surgical treatment at our hospital. The study protocol was approved by the Institutional Review Board (IRB) of the hospital (Reference number: 2018-24; Date of approval: 2 April 2018). Each participant gave written informed consent. Clinical and histopathologic information was retrospectively collected from individual's medical records. The following variables were extracted: age at diagnosis (years), menopause (yes or no), laterality (right side, left side, or bilateral), behavior (borderline or invasive), histological subtype (serous or non-serous), histological grade (G1, G2, or G3), Federation of Gynecologists and Obstetricians (FIGO) stage (I, II, III, or IV), intravascular tumor thrombus (yes or no), serum CA125 ( $<35$ or $\geq 35$ $\mathrm{U} / \mathrm{mL})$, serum CA19-9 $(<37$ or $\geq 37 \mathrm{U} / \mathrm{mL})$, serum CEA $(<5$ or $\geq 5 \mathrm{ng} / \mathrm{mL}$ ), and chemotherapy (yes or no).

\section{Follow-Up}

Follow-up was conducted at the dedicated unit in our hospital according to standard epidemiologic protocol (30). Follow-up started 6 months after the surgery and was performed by 
examinations every 3 months on an outpatient bases and/or by telephone calls. Follow-up information including overall survival (OS, in months), and disease-free survival (DFS, in months) was linked to the clinicopathological database using the unique patient ID number. All identifiable data was removed once the dataset was constructed to protect patient's privacy.

\section{Immunohistochemistry and Scoring}

The ovarian cancer tissues were obtained from the Tissue Bank of Obstetrics and Gynecology Hospital of Fudan University. The construction of tissue microarray was previously described (30). Ovarian cancer tissues were fixed in 4\% paraformaldehyde at $4{ }^{\circ} \mathrm{C}$ overnight, dehydrated by graded ethanol solutions and embedded in paraffin. The tissue sections $(5-\mu \mathrm{m})$ were deparaffinized and subjected to heat-induced antigen retrieval. The endogenous peroxidase activity was quenched after incubation in 3\% hydrogen peroxide for $10 \mathrm{~min}$. The sections were incubated with $10 \%$ goat serum for $30 \mathrm{~min}$ to block non-specific binding sites and then incubated with the primary antibodies $(1: 100)$ at $4^{\circ} \mathrm{C}$ overnight followed by secondary antibody $(1: 100)$ for $1 \mathrm{~h}$ at $37^{\circ} \mathrm{C}$. Bound antibody was then visualized using the EnVision ${ }^{\mathrm{TM}}$ Detection Systems (Dako, Glostrup, Denmark). The expression of CD44 was examined and evaluated using immunohistochemistry according to our previous protocol (30). The expression was independently reviewed by two observers (YD and $\mathrm{HZ}$ ). The immunostaining score was incorporated both staining intensity $(0=$ absent, $1=$ weak, $2=$ moderate, $3=$ strong) and percentage of positive cells $(0=0 \%, 1=1-25 \%, 2=26-50 \%, 3=51-75 \%, 4=76-100 \%$ of cells). The immunostaining score was calculated based on the proportion of stained tumor cells: $0-10 \%$ as negative $(-)$, $11-25 \%$ as slightly positive $(+), 26-50 \%$ as moderately positive $(++)$, and $51-100 \%$ as strongly positive $(+++)$. Patients with and + expression was combined as the lower expression group, and patients with ++ and +++ expression was combined as the higher expression group for analyses (30).

\section{Reagents and Antibodies}

Monoclonal antibodies to CD44, ZEB1, Caveolin-1, and GAPDH were obtained from Cell Signaling Technology (San Diego, CA, USA). CD44 construct was obtained from Asia-Vector Biotechnology (Shanghai, China). Secondary antibodies conjugated with HRP were obtained from Jackson ImmunoResearch Laboratories (West Grove, PA, USA). CD44, ZEB1, Caveolin-1 small interfering RNAs (siRNAs) were synthesized by RioBio Co. (Guangzhou, China). The target sequences of siRNA are indicated in Table S1.

\section{Cell Culture}

HO8910PM-Snail (HOPM-Snail), a stable Snail-expressing cell line, and its control cell line HO8910PM-vector (HOPM) were generated as described previously (31). All the cells were cultured in 1640 complete medium supplemented with $10 \%$ FBS and $400 \mu \mathrm{g} / \mathrm{ml}$ of $\mathrm{G} 418$ in $5 \% \mathrm{CO}_{2}$ at $37^{\circ} \mathrm{C}$.
TABLE 1 | Clinicopathological characteristics of study patients $(n=90)$.

\begin{tabular}{|c|c|c|}
\hline Variables & $N$ & $\%$ \\
\hline \multicolumn{3}{|l|}{ Age at diagnosis } \\
\hline Median (range) [years] & 51 & $26-74$ \\
\hline \multicolumn{3}{|l|}{ Age } \\
\hline$\leq 50$ years & 42 & 46.7 \\
\hline$>50$ years & 48 & 53.3 \\
\hline \multicolumn{3}{|l|}{ Overall survival } \\
\hline Median (range) [months] & 74.58 & $1.60-103.13$ \\
\hline Number of deaths & 43 & 47.8 \\
\hline \multicolumn{3}{|l|}{ Disease-free survival } \\
\hline Median (range) [months] & 67.65 & $0.80-103.13$ \\
\hline Number of recurrences & 47 & 52.2 \\
\hline \multicolumn{3}{|l|}{ Menopause } \\
\hline Yes & 45 & 50.0 \\
\hline No & 45 & 50.0 \\
\hline \multicolumn{3}{|l|}{ Laterality } \\
\hline Right side & 24 & 26.7 \\
\hline Left side & 18 & 20.0 \\
\hline Bilateral & 48 & 53.3 \\
\hline \multicolumn{3}{|l|}{ Histological subtype } \\
\hline Serous & 59 & 65.6 \\
\hline Non-serous & 31 & 34.4 \\
\hline \multicolumn{3}{|l|}{ Histological grade } \\
\hline G1 & 8 & 8.9 \\
\hline G2 & 21 & 23.3 \\
\hline G3 & 37 & 41.1 \\
\hline Missing & 24 & 26.7 \\
\hline \multicolumn{3}{|l|}{ FIGO } \\
\hline 1 & 28 & 31.1 \\
\hline$\|$ & 13 & 14.4 \\
\hline III & 44 & 48.9 \\
\hline IV & 5 & 5.6 \\
\hline \multicolumn{3}{|l|}{ Intravascular tumor thrombus } \\
\hline Yes & 16 & 17.8 \\
\hline No & 71 & 78.9 \\
\hline Missing & 3 & 3.3 \\
\hline \multicolumn{3}{|l|}{ Serum CA125 } \\
\hline$<35 \mathrm{U} / \mathrm{mL}$ & 12 & 13.3 \\
\hline$\geq 35 \mathrm{U} / \mathrm{mL}$ & 64 & 71.1 \\
\hline Missing & 14 & 15.6 \\
\hline \multicolumn{3}{|l|}{ Serum CA19-9 } \\
\hline$<37 \mathrm{U} / \mathrm{mL}$ & 39 & 43.3 \\
\hline$\geq 37 \mathrm{U} / \mathrm{mL}$ & 17 & 18.9 \\
\hline Missing & 34 & 37.8 \\
\hline \multicolumn{3}{|l|}{ Serum CEA } \\
\hline$<5 \mathrm{ng} / \mathrm{mL}$ & 60 & 66.7 \\
\hline$\geq 5 \mathrm{ng} / \mathrm{mL}$ & 2 & 2.2 \\
\hline Missing & 28 & 31.1 \\
\hline \multicolumn{3}{|l|}{ Chemotherapy } \\
\hline Yes & 86 & 95.6 \\
\hline Missing & 4 & 4.4 \\
\hline
\end{tabular}

(Continued) 
TABLE 1 | Continued

\begin{tabular}{lcc}
\hline Variables & $\boldsymbol{N}$ & $\%$ \\
\hline CD44 & & \\
- & 28 & 31.1 \\
+ & 13 & 14.4 \\
++ & 44 & 48.9 \\
+++ & 5 & 5.6 \\
\hline
\end{tabular}

CA 125, cancer antigen 125; CA 19-9, cancer antigen 19-9; CEA, carcinoembryonic antigen; FIGO, Federation of Gynecologists and Obstetricians.

\section{Transfection}

Cells were seeded in 6-well plates at a density of $10^{5} /$ well, and transfected with CD44 siRNA or scrambled siRNA using Lipo2000 reagent according to the manufacturer's instructions. At $48 \mathrm{~h}$ post-transfection, the cell pellets were harvested for western blotting, cell migration, and invasion assay. The transfection accuracy was evaluated by detecting the expression of target genes using western blotting analysis at $48 \mathrm{~h}$ after transfection.

\section{Western Blotting}

Ovarian cancer cells were lysed in $1 \times$ SDS lysis buffer $(50 \mathrm{mM}$ Tris- $\mathrm{HCl}, \mathrm{pH} 6.8,2 \%$ SDS, $10 \%$ glycerol, $1 \mathrm{mM}$ PMSF, and $1 \mathrm{mM} \mathrm{Na} 3 \mathrm{VO} 4$ ) and performed as previously described (32). An equal amount of total protein from various cell lysates was loaded on a SDS-PAGE gel and transferred to PVDF membrane (Millipore Corporation, USA). The membrane was blocked with 5\% BSA in PBS (containing 0.05\% Tween 20) and then incubated with specific primary antibodies and followed by incubation with HRP-conjugated secondary antibodies (Jackson ImmunoResearch Laboratories, West Grove, PA, USA). The protein bands of interest were visualized by fluorography using an enhanced chemiluminescence system (Thermo Fisher Scientific, USA).

\section{Cell Invasion}

Cell invasion were evaluated using transwell assay. Cells grown to $60-70 \%$ confluence were transfected with CD 44 siRNA. At $48 \mathrm{~h}$ post-transfection, cells were harvested by trypisinzation, pelleted by centrifugation, washed twice with PBS and resuspended in $0.1 \%$ fetal bovine serum in 1640 medium at a density of $5 \times 10^{5}$ cells $/ \mathrm{ml} .3 \times 10^{4}$ cells were added in the upper chamber of an insert (pore size, $8 \mu \mathrm{m}$, Costar Corporation). The lower chamber was filled with 1640 medium containing 10\% fetal bovine serum as an inducer. For invasion assay, $5 \times 10^{4}$ of cells were placed into the upper chamber precoated with Matrigel (BD Biosciences, Bedford, MA, USA). After $48 \mathrm{~h}$ incubation and removal of the cells on the upper chamber of the filters with a cotton swab, the cells that migrated to the lower surface of the filters were fixed in $4 \%$ paraformaldehyde and stained with crystal violet. Migrating cells were monitored with a LEICA microscope (Olympus IX71, Japan). Five visual fields of each insert were randomly chosen and the number of cells that migrated to the lower surface was counted for each well. The assays were performed in triplicate.

\section{Wound Healing Assay}

Cells were seeded in 6-well plates and transfected with CD44 siRNA, and grown to confluence. A linear wound was generated in the cell layer by scratching with sterile $200 \mu \mathrm{L}$ pipette tips. Cellular debris was removed by washing with medium. From each of these scratches, three representative images of the scratched areas were photographed at $48 \mathrm{~h}$ to analyze the migration capacity. The experiments were performed in triplicate.

\section{D-matrigel Culture}

The 3D matrigel-based culture system was performed according to the manufacture's instruction. Briefly, pre-cooled 8-well chamber slide was coated by matrigel $(80-100 \mu \mathrm{l} /$ well $)$ at $37^{\circ} \mathrm{C}$ for $15 \mathrm{~min} .5 \times 10^{4} / \mathrm{ml}$ cells in $4 \%$ matrigel were seeded in 6-well plate and maintained in $37^{\circ} \mathrm{C}$. The medium were changed every 3-4 days with $2 \%$ matrigel. Cell clones were monitored with a LEICA microscope (Olympus IX71, Japan).

\section{Reverse Phase Protein Array (RPPA) Analysis}

HOPM and HOPM-Snail cells were transfected with CD44 siRNA and then subjected to RPPA analysis at the University of Texas, M.D. Anderson Cancer Center RPPA Core Facility. Briefly, cells were grown in 6-well plates at a density of $10^{5} /$ well. At $48 \mathrm{~h}$ post-transfection, cells were lysed in lysis buffer ( $1 \%$ Triton X-100, $50 \mathrm{mM}$ HEPES, pH 7.4, $150 \mathrm{mM} \mathrm{NaCl}, 1.5 \mathrm{mM}$ $\mathrm{MgCl}_{2}, 1 \mathrm{mM}$ EGTA, $100 \mathrm{mM} \mathrm{NaF}, 10 \mathrm{mM} \mathrm{Na} 4 \mathrm{P}_{2} \mathrm{O}_{7} \cdot 10 \mathrm{H}_{2} \mathrm{O}$, $1 \mathrm{mM} \mathrm{Na} 3 \mathrm{VO}_{4}, 10 \%$ glycerol, containing freshly added protease and phosphatase inhibitors). The cell lysates were centrifuged and collected to determine protein concentration by BCA. Cell lysates were boiled for $5 \mathrm{~min}$, and stored in $-80^{\circ} \mathrm{C}$ until sample submission.

\section{Statistical Analysis}

Chi-square test or Fisher's exact test was used to determine the differences of clinicopathogical variables between high- and low-expression subgroups. Kaplan-Meier method was used to estimate OS and DFS, and log-rank test was used to compare the curves of different expression groups. Cox proportional hazards models were performed to estimate the survival distributions, and to calculate hazard ratios (HRs) and corresponding $95 \%$ confidence intervals ( $95 \%$ CIs). Variables that reach the statistical significance level in the univariate model were included in the multivariate analysis. For cell line studies, results were expressed as mean \pm standard error of the mean (SEM), and were evaluated using one-way ANOVA followed by the Dunnett test. All significance tests were two sided; $p$-value of $<0.05$ was considered as statistically significant. Data analyses were performed by STATA version 15 (StataCorp LLC, College Station, TX, USA). 


\section{RESULTS}

\section{Baseline Characteristics of the Study Population}

A total of 90 ovarian cancer patients were included in the current study. The clinicopahtological characteristics of the study population are presented in Table 1. The average age of these patients was $51.2 \pm 9.5$ years old. There were more patients with serous ovarian cancer $(n=59,65.6 \%)$. More than half of the patients were diagnosed at advanced stage (FIGO stages III-IV, $n=49,54.5 \%)$.

\section{Expression of CD44 in Ovarian Cancer Tissues}

The immunohistochemistry data showed that CD44 was almost undetectable in healthy ovarian tissues (Figure S1) while mainly located in the nuclei of ovarian cancer tissue. Figure 1A shows the representative immunostaining of CD44 at different FIGO stages of ovarian cancer. The expression of CD44 increased with the advancement of FIGO stage. Table 2 presents the association between CD44 expression and clinicopathological characteristics. High expression of CD44 was associated with higher histological grade $(p=0.014)$, and more advanced FIGO stage $(p=0.001)$. There was no significant association between CD44 expression and any other clinicopahtological factors.

\section{High Expression of CD44 Predicts Poor Prognosis in Ovarian Cancer Patients}

The median post-operative follow-up time was 74.58 months (range: $1.60-103.13$ months). During the time period, 43 patients died, 4 relapsed but were still alive at the end of the study. Kaplan-Meier curves of CD44 expression and OS and DFS are presented in Figures 1B,C. High expression of CD44 significantly associated with both worse OS and DFS (Log-rank test $p<0.0001$ for both). Univariate analysis results showed that advanced FIGO stage (III/IV vs. I/II, HR $=9.20,95 \% \mathrm{CI}=3.86-21.96, p<$ 0.0001 ), intravascular tumor thrombus (Yes vs. No, HR $=2.08$, $95 \%$ CI $=1.04-4.16, p=0.039)$, elevated CA125 level $(\geq 35$ $\mathrm{U} / \mathrm{mL}$ vs. $<35 \mathrm{U} / \mathrm{mL}, \mathrm{HR}=4.62,95 \% \mathrm{CI}=1.11-19.26, p=$ 0.035), and high expression of CD44 $(+++/++$ vs. $+/-$, HR $=4.30,95 \% \mathrm{CI}=2.20-8.40, p<0.0001)$ were associated with
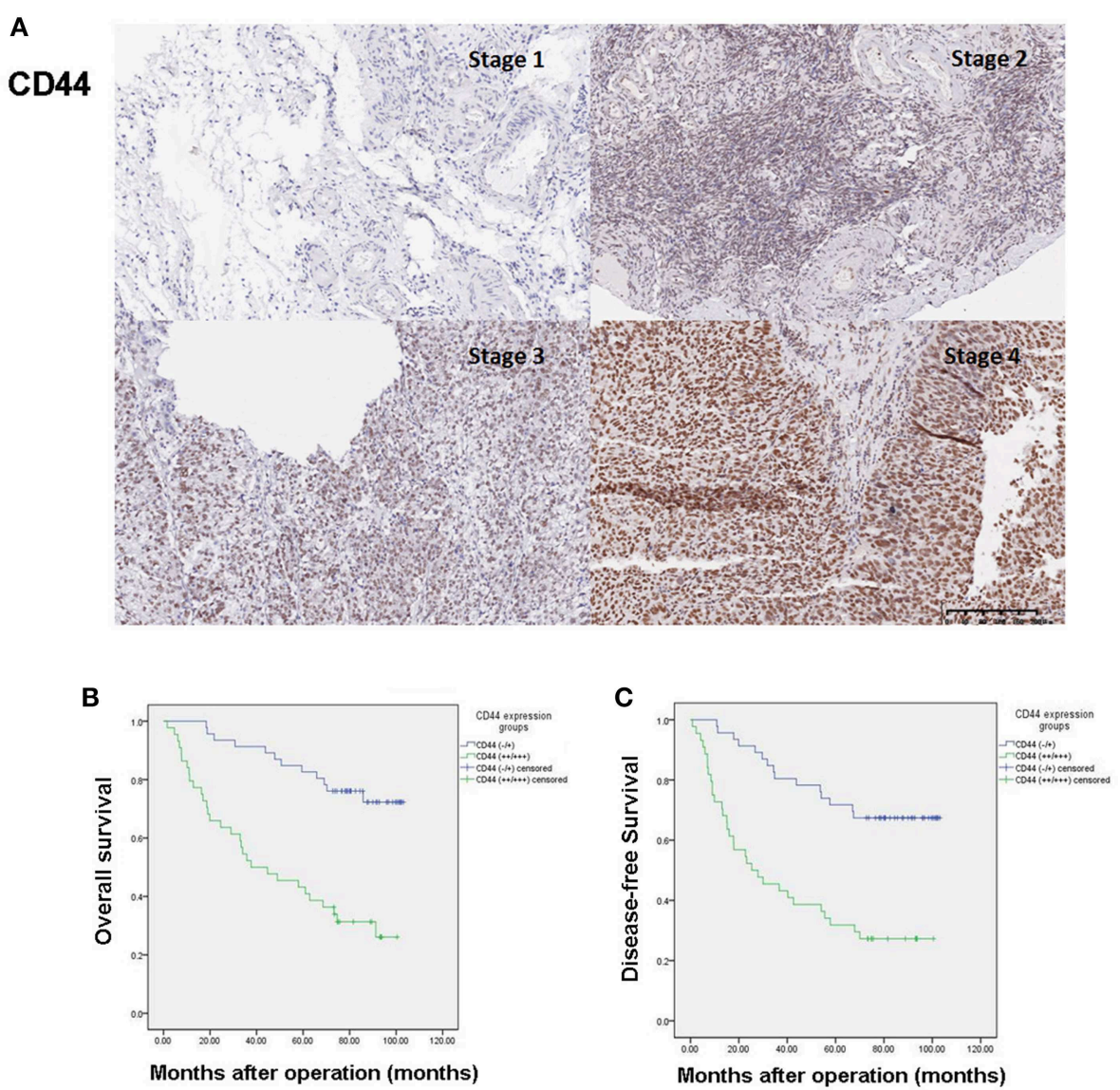

FIGURE 1 | High expression of CD44 predicts poor prognosis in ovarian cancer patients. (A) Representative immunostaining of CD44 expression at different FIGO stages of ovarian cancer. (B) High expression of CD44 is associated with poor overall survival of ovarian cancer patients. (C) High expression of CD44 is associated with poor disease-free survival of ovarian cancer patients. 
TABLE 2 | Correlation of clinicopathological characteristics with CD44 expression in 90 ovarian cancer patients.

\begin{tabular}{|c|c|c|c|}
\hline \multirow[t]{2}{*}{ Characteristics } & \multicolumn{2}{|r|}{ CD44 } & \multirow[t]{2}{*}{$p$-value } \\
\hline & $-/+(n=46)$ & $++/+++(n=44)$ & \\
\hline Age & & & 0.844 \\
\hline$\leq 50$ & 21 & 21 & \\
\hline$>50$ & 25 & 23 & \\
\hline Menopause & & & 0.673 \\
\hline Yes & 22 & 23 & \\
\hline No & 24 & 21 & \\
\hline Laterality & & & $0.142^{*}$ \\
\hline Right side & 11 & 13 & \\
\hline Left side & 13 & 5 & \\
\hline Bilateral & 22 & 26 & \\
\hline Histological subtype & & & 0.065 \\
\hline Serous & 26 & 33 & \\
\hline Non-serous & 20 & 11 & \\
\hline Histological grade & & & 0.014 \\
\hline G1/G2 & 19 & 10 & \\
\hline G3 & 13 & 24 & \\
\hline FIGO stage & & & 0.001 \\
\hline $1 / 11$ & 29 & 12 & \\
\hline III/IV & 17 & 32 & \\
\hline Intravascular tumor thrombus & & & 0.615 \\
\hline No & 35 & 36 & \\
\hline Yes & 9 & 7 & \\
\hline Serum CA125 [U/mL] & & & 0.059 \\
\hline$<35 \mathrm{U} / \mathrm{mL}$ & 9 & 3 & \\
\hline$\geq 35 \mathrm{U} / \mathrm{mL}$ & 27 & 35 & \\
\hline Serum CA19-9 [U/mL] & & & 0.771 \\
\hline$<37$ & 20 & 19 & \\
\hline$\geq 37$ & 8 & 9 & \\
\hline Serum CEA [ng/mL] & & & $0.184^{*}$ \\
\hline$<5$ & 29 & 31 & \\
\hline$\geq 5$ & 0 & 2 & \\
\hline
\end{tabular}

${ }^{*}$ Fisher's exact test. Bold values indicate $p$-value $<0.05$.

worse OS; while higher histological subtype (serous vs. nonserous, $\mathrm{HR}=1.99,95 \% \mathrm{CI}=1.01-3.92, p=0.046$ ), advanced FIGO stage (III/IV vs. I/II, HR $=11.36,95 \% \mathrm{CI}=4.78-$ 27.03, $p<0.0001)$, elevated CA125 level $(\geq 35 \mathrm{U} / \mathrm{mL}$ vs. $<35$ $\mathrm{U} / \mathrm{mL}, \mathrm{HR}=4.93,95 \% \mathrm{CI}=1.19-20.44, p=0.028)$, and high expression of CD44 $(+++/++$ vs. $+/-$, HR $=3.57,95 \%$ $\mathrm{CI}=1.93-6.62, p<0.0001)$ were associated with worse DFS (Table 3). Multivariate analysis results showed that advanced FIGO stage (III/IV vs. I/II, HR $=11.61,95 \% \mathrm{CI}=2.47-54.60$, $p=0.002)$ and high expression of CD44 $(+++/++$ vs. $+/-$, $\mathrm{HR}=3.45,95 \% \mathrm{CI}=1.48-8.04, p=0.004)$ were independent prognostic factors of poor OS; and advanced FIGO stage (III/IV vs. I/II, $\mathrm{HR}=15.53,95 \% \mathrm{CI}=3.26-74.12, p=0.001)$ and high expression of CD44 $(+++/++$ vs. $+/-$, HR $=2.45,95 \%$ $\mathrm{CI}=1.12-5.37, p=0.025)$ also independently predicted worse DFS (Table 3).

\section{CD44 Knockdown by Small Silencing RNA Abrogated Both Basal Snail Expression and TGF- $\beta 1$ Induced Snail Expression in HOPM and HOPM-Snail Cells}

Although HOPM is a highly metastatic ovarian cancer cell line, its endogenous expression of Snail is low, so that it is difficult to observe the effect of CD44 on Snail expression using this cell line. Therefore, Snail high-expression cell line HOPM-Snail was used to observe the regulation of CD44 on Snail expression. Both HOPM and HOPM-Snail cells were transfected with CD44 siRNAs, Snail expression were then detected by western blotting assay. As indicated in Figures $\mathbf{2 A}, \mathbf{B}$, both siRNAs effectively suppressed CD44 expression. CD44 siRNAs, especially siRNA2 dramatically abrogated Snail expression in HOPM and HOPMSnail cells.

As TGF- $\beta 1$ has been proven to induce Snail expression, we then detected whether CD44 siRNA abrogated TGF- $\beta 1$-induced Snail expression. As shown in Figure 2C, TGF- $\beta 1$ promoted Snail expression, which can be dramatically blocked by CD44 siRNAs. Taken together, these data indicated that CD44 may be crucial for the TGF- $\beta 1$-induced Snail expression.

\section{CD44 Knockdown Caused a Decrease in ZEB1 and an Increase in Caveolin-1 Expression}

RPPA assay was then performed to elucidate the downstream targets of CD44. These data indicated that Cavolin-1 may be the possible downstream targets of CD44 (Figure 3A). Western blotting data confirmed that CD44 siRNAs caused an increase in Caveolin-1 expression, indicating that CD44 may inhibit the expression of Caveolin-1 (Figure 3B).

Considering the crucial role of Snail in EMT, we hypothesized that CD44 may regulate the EMT process. Next, we examined the effect of CD44 on the other EMT inducers. Our findings showed that CD44 siRNA caused a decrease in ZEB1 expression in HOPM and HOPM-Snail cells, indicating that CD44 may regulate ZEB1 expression (Figure 3B).

We further detected whether ZEB1 exerts a negative feedback regulation on CD44. The HOPM and HOPM-Snail cells were treated with ZEB1 siRNAs, and the expression of CD44 in these cells was detected by western blot analysis. As shown in Figure 3C, ZEB1 siRNAs exerted no effects in CD44 expression, indicating that ZEB1 could not regulate CD44 expression negatively. Similarly, we also detected whether Caveolin1 modulated CD44 expression (Figure 3C). As shown in Figure 3D, HOPM and HOPM-Snail cells treated with Caveolin1 siRNAs demonstrated similar CD44 expression compared with their control counterparts, indicating that there is no reciprocal regulation between Caveolin-1 and CD44.

\section{CD44 Knockdown Resulted in Reduced Cell Migration, Cell Invasion, and Clone Formation in HOPM and HOPM-Snail Cells}

We then observed the effect of CD44 siRNA on the growth, migration and invasion of ovarian cancer. 3D matrigel culture 
TABLE 3 | Univariate and multivariate analyses of factors associated with overall survival.

\begin{tabular}{|c|c|c|c|c|}
\hline \multirow[t]{2}{*}{ Variables } & \multicolumn{2}{|c|}{ Overall survival } & \multicolumn{2}{|c|}{ Disease-free survival } \\
\hline & HR $(95 \% \mathrm{Cl})$ & $p$-value & HR (95\% Cl) & $p$-value \\
\hline \multicolumn{5}{|l|}{ Univariate analyses } \\
\hline Age (>50 vs. $\leq 50)$ & $0.97(0.53-1.76)$ & 0.913 & $0.93(0.52-1.64)$ & 0.793 \\
\hline Histological subtype (serous vs. non-serous) & $1.94(0.96-3.95)$ & 0.066 & $1.99(1.01-3.92)$ & 0.046 \\
\hline FIGO stage (III/IV vs. I/II) & $9.20(3.86-21.96)$ & $<0.0001$ & $11.36(4.78-27.03)$ & $<0.0001$ \\
\hline Histological grade (G1/G2 vs. G3) & $1.52(0.76-3.03)$ & 0.238 & $1.58(0.81-3.07)$ & 0.181 \\
\hline Intravascular tumor thrombus (Yes vs. No) & $2.08(1.04-4.16)$ & 0.039 & $1.86(0.94-3.69)$ & 0.074 \\
\hline CA125 ( $\geq 35 \mathrm{U} / \mathrm{mL}$ vs. $<35 \mathrm{U} / \mathrm{mL})$ & $4.62(1.11-19.26)$ & 0.035 & $4.93(1.19-20.44)$ & 0.028 \\
\hline CD44 $(+++/++$ vs. $+/-)$ & $4.30(2.20-8.40)$ & $<0.0001$ & 3.57 (1.93-6.62) & $<0.0001$ \\
\hline \multicolumn{5}{|l|}{ Multivariate analyses } \\
\hline FIGO stage (III/IV vs. I/II) & $11.61(2.47-54.60)$ & 0.002 & $15.53(3.26-74.12)$ & 0.001 \\
\hline CD44 $(+++/++$ vs. $+/-)$ & $3.45(1.48-8.04)$ & 0.004 & $2.45(1.12-5.37)$ & 0.025 \\
\hline
\end{tabular}

CA 125, cancer antigen 125; FIGO, Federation of Gynecologists and Obstetricians; HR, hazard ratio; 95\% Cl, 95\% confidence interval. Bold values indicate p-value < 0.05.

system confirmed that CD44 siRNA abrogated the growth of HOPM and HOPM-Snail cells (Figure 4A). Wound healing assay and transwell showed that CD44 siRNA abrogated cell migration and invasion capacities of HOPM and HOPM-Snail cells (Figures 4B-D). These data indicated that CD44 may be crucial for the growth, migration, and invasion of human ovarian cancer. We also evaluated the expression of CD44 in a series of ovarian cancer cell lines with different invasive potential (Figure S2). CD44 expression in SKOV3 and SKOV3ip cells seemed to be consistent with their invasive capacities as CD44 expression in SKOV3ip is much higher than that in SKOV3 cells. However, CD44 expression in the high metastatic HOPM-Snail cells was not higher than that in HOPM cells. The high metastatic ovarian cancer cell line OVCAR-5 did not even demonstrate CD44 expression. Therefore, although CD44 is crucial for the invasion of ovarian cancer, it may not be the only determinant of invasive capacity of different ovarian cancer cell lines.

\section{DISCUSSION}

In the present study, we demonstrated that CD44 expression positively correlated with FIGO stage and histological grade of ovarian cancer. We observed that up-regulation of CD44 expression was an independent prognostic factor of both poor OS and DFS. Furthermore, our in vitro data suggested that CD44 may promote ovarian cancer progression through the EMT process by regulating Snail and ZEB1. Our study suggested that CD44 may serve as a prognostic marker as well as possible treatment target for ovarian cancer.

A number of studies have been conducted to investigate the association between CD44 expression and ovarian cancer prognosis, with controversial results $(8-18,33,34)$. Similar to our results, studies reported that ovarian cancer patients with positive expression of CD44 variant had a significantly shorter DFS (8), while low levels of CD44 expression was associated with better survival (12). Another study conducted in the US also reported that the expression of standard CD44 (CD44s) was significantly

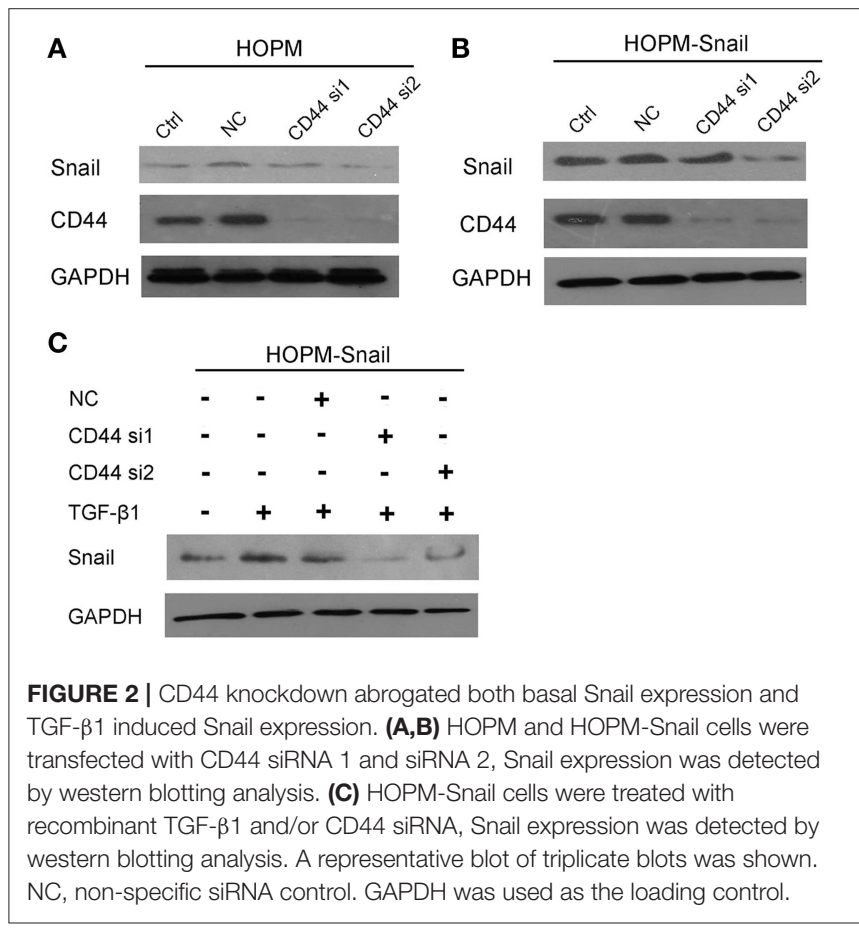

associated with worse DFS both in univariate $(p=0.003)$ and multivariate $(p=0.006)$ analysis in 56 patients with epithelial ovarian cancer (9). A study from Korea also reported that over expression of CD44s was an independent prognostic factor of lower overall survival rate (17). In addition, expression of CD44 spliced variant $6(\mathrm{CD} 44 \mathrm{v} 6)$ was reported to be associated with a shortened overall survival in stage III-IV patients as well as the recurrence of ovarian serous cancer $(10,11)$. A more recent meta-analysis pooling data from 2,161 patients further reported that positive expression of CD44 was significantly associated with poor 5 -year overall survival $(\mathrm{RR}=1.42,95 \% \mathrm{CI}$ : $1.01-2.00, p=$ 0.05) (18). 


\section{A}

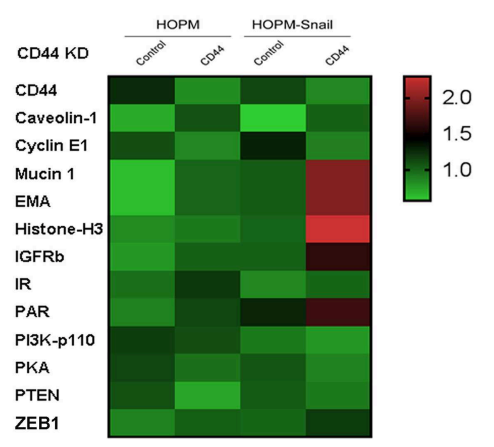

C

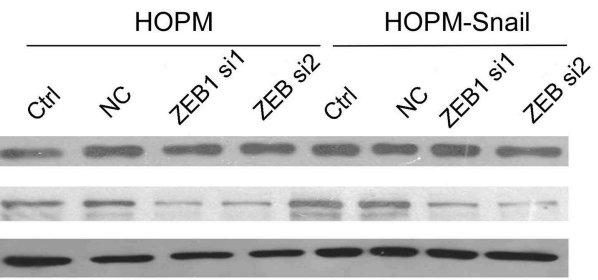

B

Caveolin-1

ZEB1

CD44

GAPDH
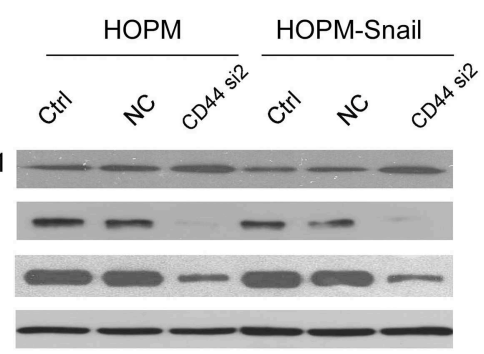

D

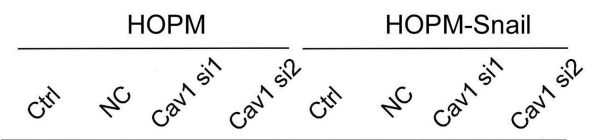

CD44

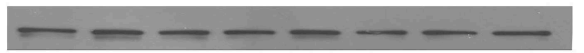

Caveolin-1

GAPDH

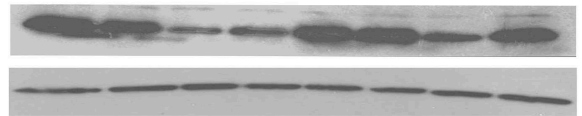

FIGURE 3 | CD44 knockdown caused a decrease in ZEB1 expression and increase in Caveolin-1 expression. (A) RPPA analysis of the differential gene expression in HOPM and HOPM-Snail cells transfected with CD44 siRNAs. (B) HOPM and HOPM-Snail cells were transfected with CD44 siRNA, the expression of ZEB1 and Caveolin-1 was detected by western blotting analysis. (C) HOPM and HOPM-Snail cells were transfected with ZEB1 siRNA, CD44 expression was detected by western blotting analysis. (D) HOPM and HOPM-Snail cells were transfected with Caveolin-1 siRNA, CD44 expression was detected by western blotting analysis. A representative blot of triplicate blots was shown. NC, non-specific siRNA control. GAPDH was used as the loading control.
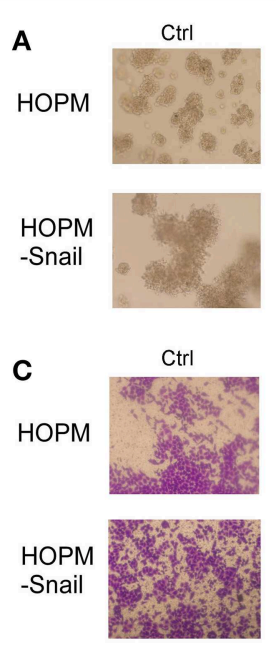

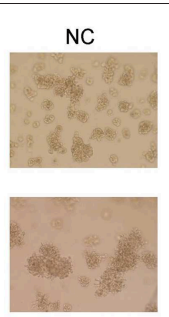

NC

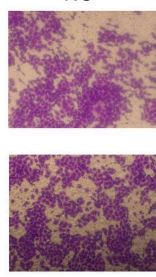

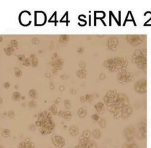

B
HOPM

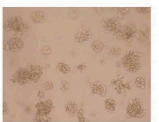

HOPM
-Snail

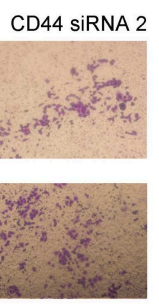

D
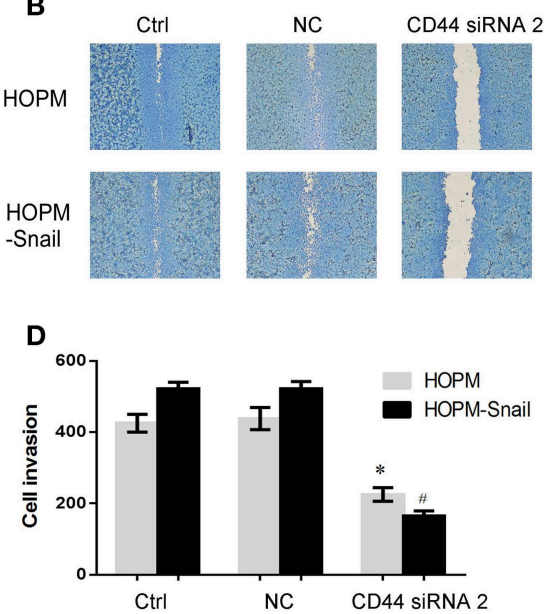

FIGURE 4 | CD44 knockdown resulted in reduced clone formation, cell migration, and cell invasion of HOPM and HOPM-Snail cells. (A) HOPM and HOPM-Snail cells were transfected with CD44 siRNA 2, clone formation capacity was detected by 3-D culture assay. (A) At $48 \mathrm{~h}$ post-transfection, cells in $4 \%$ matrigel were seeded in 6-well plate and maintained in $37^{\circ} \mathrm{C}$ for 7-14 days, formed cell clones were then photographed. A representative image of triplicate experiments was shown. (B) HOPM and HOPM-Snail cells were transfected with CD44 siRNA 2, cell migration was detected by wound healing assay. At 48h post-transfection, a linear wound was generated in the cells with 70-80\% confluence and the scratched areas were photographed. A representative image of triplicate experiments was shown. (C,D) HOPM and HOPM-Snail cells were transfected with CD44 siRNA 2, cell invasion was detected by transwell assay. At 48 h post-transfection, cells were plated in the upper chamber of an insert and the cells that migrated to the lower surface of the filters were monitored after $48 \mathrm{~h}$ incubation. A representative image (C) and quantitative analysis (D) were shown. ${ }^{*} P<0.05$ compared with HOPM control cells. $\# P<0.05$ compared with HOPM-Snail control cells. NC, non-specific siRNA control.

However, there are also studies that did not find any association between CD44 variant expression and survival $(13,33)$. Some studies also reported that down-regulation of
CD44 is associated with unfavorable prognosis (14-16). Loss of CD44s expression was associated with shorter survival, although the association did not maintain statistical significance 
after multivariate adjustment (15). Some studies showed that expression of CD44 was an independent predictor of favorable 5 -year recurrence-free survival (16). More interestingly, there is also research showing that the expression of CD44 splice variant and the survival of ovarian cancer is site dependent (34). Expression of CD44-v10 in metastatic lesions was associated with decreased survival, while expression of CD44s in the primary tumor and the tumor-stroma interface was associated with improved survival (34). These conflicting findings may due to inadequate sample size and/or power, or different splicing variants of CD44.

EMT is a process by which cells gain the ability to escape from the epithelial layer and invade secondary sites, and form metastases (25). Previous data have shown that CD44 may regulate EMT progress by regulating E-cadherin, vimentin, and $\mathrm{N}$-cadherin expression (29). In addition, CD44s was up-regulated upon TGF- $\beta 1$-induced EMT. Moreover, CD44s over-expression in ovarian cancer cells induced EMT and caused gain of stemlike features and chemoresistance (29). These data indicated that CD44 may regulate the EMT process. On the basis of previous studies, our findings further demonstrated that CD44 siRNAs lead to the decrease of Snail and ZEB1, indicating that CD44 may promote the EMT process through Snail and ZEB1. Snail has been well-characterized as the inducer of EMT and its expression is sufficient for the initiation of EMT (35). ZEB1 is another crucial regulator of EMT. The activation of ZEB1 and Snail has been reported to, either directly or indirectly represses the Ecadherin $(\mathrm{CDH} 1)$ promoter and drives the EMT process $(35,36)$. Therefore, our data has elucidated a new mechanism by which CD44 induces the EMT progress.

In addition, our data demonstrated that CD44 knockdown lead to an increase of Caveolin-1. Caveolin-1 is a scaffolding protein which promotes the biogenesis of caveolae (37). It has been reported that Caveolin-1 acts as a tumor suppressor in ovarian and breast cancers, and high expression of Caveolin-1 may correlate with favorable outcome of ovarian cancer (38-40). Presumably, up-regulation of Caveolin-1 in CD44 knockdown ovarian cancer cells may partially contribute to the favorable prognosis of ovarian cancer patients.

Chemotherapy resistance is a main reason of therapeutic failure and poor prognosis of ovarian cancer patients, which becomes a major obstacle of ovarian cancer treatment (5). CD44, as a cancer stem cell marker, also plays a pivotal role in chemoresistance. Recent studies have shown that CD44 knockdown increases the sensitivity to anticancer drugs in breast cancer cells, myeloma, and colon cancer (41-44). Our in vitro data further suggested that CD44 silencing caused a decrease in the ZEB1 expression. Notably, down-regulation of ZEB1 resulted in significant inhibition of cisplatin-resistance in ovarian and lung cancers $(45,46)$. Presumably, CD44 may regulate chemoresistance of ovarian cancer via ZEB1. Since we do not have chemoresistance data due to difficulties in clinical follow-up, we could not assess the association between CD44 expression and chemotherapy failure in the present study. However, most of the ovarian cancer patients die due to recurrence after development of chemoresistance. In the future, well-designed cohort studies with a larger sample size and more complete follow-up data, as well as in-depth molecular mechanism researches are needed to elucidate the role of CD44 and ZEB1 in the chemoresistance of ovarian cancer.

Taken together, our present study indicated that CD44 may be pivotal for EMT and closely correlate with the prognosis of ovarian cancer. Clarifying the role of CD44 in the prognosis of ovarian cancer may be of great help for the development of novel treatment strategies.

\section{CONCLUSIONS}

In conclusion, our study suggested that high expression of CD44 was correlated with poor prognosis of ovarian cancer patients. Our data also showed that ZEB1, Snail, and Caveolin-1 are regulated by $\mathrm{CD} 44$, indicating the crucial role of CD44 in the initiation of EMT in ovarian cancer. Presumably, CD44 may be a potent therapeutic target of human ovarian cancer.

\section{DATA AVAILABILITY}

The datasets generated for this study are available on request to the corresponding author.

\section{AUTHOR CONTRIBUTIONS}

HZ, YY, and CX conceived and designed study. JZ, BL, and YY performed the cell line studies. YD and HZ wrote the manuscript and assessed the IHC score. YL performed the RPPA analysis. YD reviewed the clinical records and conducted the statistical analysis. All authors read and approved the final manuscript.

\section{FUNDING}

This work was funded by the National Key R\&D Program of China (Grant Number: 2016YFC1303100), Shanghai Talent Development Fund (Grant Number: 2017090), and National Natural Science Foundation of China (Grant number: 81571457).

\section{SUPPLEMENTARY MATERIAL}

The Supplementary Material for this article can be found online at: https://www.frontiersin.org/articles/10.3389/fonc. 2019.00802/full\#supplementary-material

Figure S1 | CD44 was almost undetectable in normal ovarian tissues. Immunohistochemistry analysis of CD44 expression in four normal ovarian tissues from patients with benign disease who underwent hysterectomy and bilateral appendectomy. Scale bar $=200 \mu \mathrm{M}$.

Figure S2 | CD44 expression may not necessarily be associated with the invasive potential of different ovarian cancer cell lines. Western blotting analysis of CD44 expression in HOPM, HOPM-Snail, Skov3, Skov3ip, OC316, Hey, ES2, OVCAR-3, OVCAR-5 cell lines. GAPDH was used as the loading control. 


\section{REFERENCES}

1. Bray F, Ferlay J, Soerjomataram I, Siegel RL, Torre LA, Jemal A. Global cancer statistics 2018: GLOBOCAN estimates of incidence and mortality worldwide for 36 cancers in 185 countries. CA Cancer J Clin. (2018) 68:394-424. doi: $10.3322 /$ caac. 21492

2. Chen W, Zheng R, Baade PD, Zhang S, Zeng H, Bray F, et al. Cancer statistics in China, 2015. CA Cancer J Clin. (2016) 66:115-32. doi: 10.3322/caac.21338

3. Mørch LS, Løkkegaard E, Andreasen AH, Kjaer SK, Lidegaard O. Hormone therapy and different ovarian cancers: a national cohort study. Am J Epidemiol. (2012) 175:1234-42. doi: 10.1093/aje/kwr446

4. Hunn J, Rodriguez GC. Ovarian cancer: etiology, risk factors, and epidemiology. Clin Obstet Gynecol. (2012) 55:3-23. doi: 10.1097/ GRF.0b013e31824b4611

5. Eisenhauer EA. Real-world evidence in the treatment of ovarian cancer. Ann Oncol. (2017) 28(Suppl. 8):viii61-5. doi: 10.1093/annonc/mdx443

6. Yan Y, Zuo X, Wei D. Concise review: emerging role of CD44 in cancer stem cells: a promising biomarker and therapeutic target. Stem Cells Transl Med. (2015) 4:1033-43. doi: 10.5966/sctm.2015-0048

7. Thapa R, Wilson GD. The importance of CD44 as a stem cell biomarker and therapeutic target in cancer. Stem Cells Int. (2016) 2016:2087204. doi: $10.1155 / 2016 / 2087204$

8. Uhl-Steidl M, Müller-Holzner E, Zeimet AG, Adolf GR, Daxenbichler G, Marth C, et al. Prognostic value of CD44 splice variant expression in ovarian cancer. Oncology. (1995) 52:400-6. doi: 10.1159/0002 27497

9. Kayastha S, Freedman AN, Piver MS, Mukkamalla J, Romero-Guittierez M, Werness BA. Expression of the hyaluronan receptor, CD44S, in epithelial ovarian cancer is an independent predictor of survival. Clin Cancer Res. (1999) 5:1073-6.

10. Shi J, Zhou Z, Di W, Li N. Correlation of CD44v6 expression with ovarian cancer progression and recurrence. BMC Cancer. (2013) 13:182. doi: 10.1186/1471-2407-13-182

11. Tjhay F, Motohara T, Tayama S, Narantuya D, Fujimoto K, Guo J, et al. CD44 variant 6 is correlated with peritoneal dissemination and poor prognosis in patients with advanced epithelial ovarian cancer. Cancer Sci. (2015) 106:14218. doi: $10.1111 /$ cas. 12765

12. Wang H, Tan M, Zhang S, Li X, Gao J, Zhang D, et al. Expression and significance of CD44, CD47 and c-met in ovarian clear cell carcinoma. Int $J$ Mol Sci. (2015) 16:3391-404. doi: 10.3390/ijms16023391

13. Cannistra SA, Abu-Jawdeh G, Niloff J, Strobel T, Swanson L, Andersen J, et al. CD44 variant expression is a common feature of epithelial ovarian cancer: lack of association with standard prognostic factors. J Clin Oncol. (1995) 13:1912-21. doi: 10.1200/JCO.1995.13.8.1912

14. Saegusa M, Machida D, Hashimura M, Okayasu I. CD44 expression in benign, premalignant, and malignant ovarian neoplasms: relation to tumour development and progression. J Pathol. (1999) 189:32637. doi: 10.1002/(SICI)1096-9896(199911)189:3<326::AID-PATH425>3.0. $\mathrm{CO} ; 2-6$

15. Ross JS, Sheehan CE, Williams SS, Malfetano JH, Szyfelbein WM, Kallakury BV. Decreased CD44 standard form expression correlates with prognostic variables in ovarian carcinomas. Am J Clin Pathol. (2001) 116:122-8. doi: 10.1309/KUK0-1M3D-LGNE-THXR

16. Sillanpää S, Anttila MA, Voutilainen K, Tammi RH, Tammi MI, Saarikoski $\mathrm{SV}$, et al. CD44 expression indicates favorable prognosis in epithelial ovarian cancer. Clin Cancer Res. (2003) 9:5318-24.

17. Cho EY, Choi Y, Chae SW, Sohn JH, Ahn GH. Immunohistochemical study of the expression of adhesion molecules in ovarian serous neoplasms. Pathol Int. (2006) 56:62-70. doi: 10.1111/j.1440-1827.2006. 01925.x

18. Lin J, Ding D. The prognostic role of the cancer stem cell marker CD44 in ovarian cancer: a meta-analysis. Cancer Cell Int. (2017) 17:8. doi: 10.1186/s12935-016-0376-4

19. Zeimet AG, Reimer D, Sopper S, Boesch M, Martowicz A, Roessler J, et al. Ovarian cancer stem cells. Neoplasma. (2012) 59:747-55. doi: 10.4149/neo_2012_094

20. Morrison R, Schleicher SM, Sun Y, Niermann KJ, Kim S, Spratt DE, et al. Targeting the mechanisms of resistance to chemotherapy and radiotherapy with the cancer stem cell hypothesis. J Oncol. (2011) 2011:941876. doi: 10.1155/2011/941876

21. Patrawala L, Calhoun T, Schneider-Broussard R, Li H, Bhatia B, Tang S, et al. Highly purified CD44+ prostate cancer cells from xenograft human tumors are enriched in tumorigenic and metastatic progenitor cells. Oncogene. (2006) 25:1696-708. doi: 10.1038/sj.onc. 1209327

22. Takaishi S, Okumura T, Tu S, Wang SS, Shibata W, Vigneshwaran R, et al. Identification of gastric cancer stem cells using the cell surface marker CD44. Stem Cells. (2009) 27:1006-20. doi: 10.1002/stem.30

23. Du L, Wang H, He L, Zhang J, Ni B, Wang X, et al. CD44 is of functional importance for colorectal cancer stem cells. Clin Cancer Res. (2008) 14:675160. doi: 10.1158/1078-0432.CCR-08-1034

24. Zhang J, Yuan B, Zhang H, Li H. Human epithelial ovarian cancer cells expressing CD105, CD44 and CD106 surface markers exhibit increased invasive capacity and drug resistance. Oncol Lett. (2019) 17:5351-60. doi: 10.3892/ol.2019.10221

25. Nieto MA, Huang RY, Jackson RA, Thiery JP. EMT: 2016. Cell. (2016) 166:21-45. doi: 10.1016/j.cell.2016.06.028

26. Mitra R, Chen X, Greenawalt EJ, Maulik U, Jiang W, Zhao Z, et al. Decoding critical long non-coding RNA in ovarian cancer epithelial-to-mesenchymal transition. Nat Commun. (2017) 8:1604. doi: 10.1038/s41467-01701781-0

27. Lu W, Kang Y. Epithelial-mesenchymal plasticity in cancer progression and metastasis. Dev Cell. (2019) 49:361-74. doi: 10.1016/j.devcel.2019.04.010

28. Keshamouni VG, Schiemann WP. Epithelial-mesenchymal transition in tumor metastasis: a method to the madness. Future Oncol. (2009) 5:1109-11. doi: $10.2217 /$ fon. 09.87

29. Bhattacharya R, Mitra T, Ray Chaudhuri S, Roy SS. Mesenchymal splice isoform of CD44 (CD44s) promotes EMT/invasion and imparts stem-like properties to ovarian cancer cells. J Cell Biochem. (2018) 119:3373-83. doi: $10.1002 / j c b .26504$

30. Du Y, Tao X, Wu J, Yu H, Yu Y, Zhao H. APOBEC3B up-regulation independently predicts ovarian cancer prognosis: a cohort study. Cancer Cell Int. (2018) 18:78. doi: 10.1186/s12935-018-0572-5

31. Jia L, Jin H, Zhou J, Chen L, Lu Y, Ming Y, et al. A potential anti-tumor herbal medicine, Corilagin, inhibits ovarian cancer cell growth through blocking the TGF- $\beta$ signaling pathways. BMC Complement Altern Med. (2013) 13:33. doi: 10.1186/1472-6882-13-33

32. Zhao HB, Tang CL, Hou YL, Xue LR, Li MQ, Du MR, et al. CXCL12/CXCR4 axis triggers the activation of EGF receptor and ERK signaling pathway in CsA-induced proliferation of human trophoblast cells. PLoS ONE. (2012) 7:e38375. doi: 10.1371/journal.pone.0038375

33. Elzarkaa AA, Sabaa BE, Abdelkhalik D, Mansour H, Melis M, Shaalan W, et al. Clinical relevance of CD44 surface expression in advanced stage serous epithelial ovarian cancer: a prospective study. J Cancer Res Clin Oncol. (2016) 142:949-58. doi: 10.1007/s00432-016-2116-5

34. Rodríguez-Rodríguez L, Sancho-Torres I, Mesonero C, Gibbon DG, Shih WJ, Zotalis G. The CD44 receptor is a molecular predictor of survival in ovarian cancer. Med Oncol. (2003) 20:255-63. doi: 10.1385/MO:2 0:3:255

35. Cano A, Pérez-Moreno MA, Rodrigo I, Locascio A, Blanco MJ, del Barrio MG, et al. The transcription factor snail controls epithelial-mesenchymal transitions by repressing E-cadherin expression. Nat Cell Biol. (2000) 2:76-83. doi: $10.1038 / 35000025$

36. Hou P, Li L, Chen F, Chen Y, Liu H, Li J, et al. PTBP3-mediated regulation of ZEB1 mRNA stability promotes epithelial-mesenchymal transition in breast cancer. Cancer Res. (2018) 78:387-98. doi: 10.1158/0008-5472.CAN-17-0883

37. Senetta R, Stella G, Pozzi E, Sturli N, Massi D, Cassoni P. Caveolin-1 as a promoter of tumour spreading: when, how, where and why. J Cell Mol Med. (2013) 17:325-36. doi: 10.1111/jcmm.12030

38. Sanna E, Miotti S, Mazzi M, De Santis G, Canevari S, Tomassetti A. Binding of nuclear caveolin-1 to promoter elements of growth-associated genes in ovarian carcinoma cells. Exp Cell Res. (2007) 313:1307-17. doi: 10.1016/j.yexcr.2007.02.005

39. Wiechen K, Diatchenko L, Agoulnik A, Scharff KM, Schober H, Arlt K, et al. Caveolin-1 is down-regulated in human ovarian carcinoma and acts as a candidate tumor suppressor gene. Am J Pathol. (2001) 159:1635-43. doi: $10.1016 / \mathrm{S} 0002-9440(10) 63010-6$ 
40. Du C, Chen L, Zhang H, Wang Z, Liu W, Xie X, et al. Caveolin-1 limits the contribution of BKCa channel to MCF-7 breast cancer cell proliferation and invasion. Int J Mol Sci. (2014) 15:20706-22. doi: 10.3390/ijms151 120706

41. Hiscox S, Baruha B, Smith C, Bellerby R, Goddard L, Jordan N, et al. Overexpression of CD44 accompanies acquired tamoxifen resistance in MCF7 cells and augments their sensitivity to the stromal factors, heregulin and hyaluronan. BMC Cancer. (2012) 12:458. doi: 10.1186/1471-2407-12-458

42. Boulbes DR, Chauhan GB, Jin Q, Bartholomeusz C, Esteva FJ. CD44 expression contributes to trastuzumab resistance in HER2-positive breast cancer cells. Breast Cancer Res Treat. (2015) 151:501-13. doi: 10.1007/s10549-015-3414-3

43. Bjorklund CC, Baladandayuthapani V, Lin HY, Jones RJ, Kuiatse I, Wang H, et al. Evidence of a role for CD44 and cell adhesion in mediating resistance to lenalidomide in multiple myeloma: therapeutic implications. Leukemia. (2014) 28:373-83. doi: 10.1038/leu.2013.174

44. Bates RC, Edwards NS, Burns GF, Fisher DE. A CD44 survival pathway triggers chemoresistance via lyn kinase and phosphoinositide 3-kinase/Akt in colon carcinoma cells. Cancer Res. (2001) 61:5275-83.
45. Cui Y, Qin L, Tian D, Wang T, Fan L, Zhang $\mathrm{P}$, et al. ZEB1 promotes chemoresistance to cisplatin in ovarian cancer cells by suppressing SLC3A2. Chemotherapy. (2018) 63:262-71. doi: 10.1159/000 493864

46. Ren J, Chen Y, Song H, Chen L, Wang R. Inhibition of ZEB1 reverses EMT and chemoresistance in docetaxel-resistant human lung adenocarcinoma cell line. J Cell Biochem. (2013) 114:1395-403. doi: 10.1002/jcb.24481

Conflict of Interest Statement: The authors declare that the research was conducted in the absence of any commercial or financial relationships that could be construed as a potential conflict of interest.

Copyright (C) 2019 Zhou, Du, Lu, Luan, Xu, Yu and Zhao. This is an open-access article distributed under the terms of the Creative Commons Attribution License (CC $B Y)$. The use, distribution or reproduction in other forums is permitted, provided the original author(s) and the copyright owner(s) are credited and that the original publication in this journal is cited, in accordance with accepted academic practice. No use, distribution or reproduction is permitted which does not comply with these terms. 